\title{
Ion emission from a metal surface through a multiphoton process and optical field ionization
}

\author{
Masaki Hashida, Shin Namba, Kiminori Okamuro, Shigeki Tokita, and Shuji Sakabe \\ ARCBS, Institute for Chemical Research, Kyoto University, Gokasho, Uji, Kyoto 611-0011, Japan \\ and Department of Physics, Graduate School of Science, Kyoto University, Kitashirakawa, Sakyo, Kyoto 606-7501, Japan
}

(Received 24 September 2009; revised manuscript received 24 February 2010; published 23 March 2010)

\begin{abstract}
In order to investigate the physics of ion emission under an intense optical field, the ions emitted from a laser-irradiated copper surface were studied by time-of-flight energy spectroscopy. The lowest laser fluence at which ions are emitted, $F_{t h, L}$, is $0.028 \mathrm{~J} / \mathrm{cm}^{2}$, and two higher emission thresholds were identified at fluences of $F_{t h, M}=0.195 \mathrm{~J} / \mathrm{cm}^{2}$ and $F_{t h, H}=0.470 \mathrm{~J} / \mathrm{cm}^{2}$. The relation between the number of emitted ions per pulse $N_{i}$ and the laser fluence $F$ was in good agreement with $N_{i} \propto F^{4}$ for $F_{t h, L}-F_{t h, M}, N_{i} \propto F^{3}$ for $F_{t h, M}-F_{t h, H}$, and $N_{i}$ $\propto F^{2}$ for $\geq F_{t h, H}$. The dependence of ion production on laser energy fluence is explained well by multiphoton absorption and optical field ionization. Even at a low laser fluence such as $0.136 \mathrm{~J} / \mathrm{cm}^{2}$, the emitted ions have an energy of $30 \mathrm{eV}$, and the ion energy depends on the laser fluence $\left(790 \mathrm{eV}\right.$ at $\left.14.4 \mathrm{~J} / \mathrm{cm}^{2}\right)$. The laser fluence dependence of ion energy is reasonably well related to those of the interspaces of gratings that are selforganized on a metal surface by femtosecond laser pulses.
\end{abstract}

DOI: 10.1103/PhysRevB.81.115442

PACS number(s): 79.70.+q, 79.20.Ds, 78.20.Jq

\section{INTRODUCTION}

The physics of the interaction between a solid surface and an intense optical field has been studied since the appearance of intense femtosecond lasers. Atomic and/or ion emissions from metal have been investigated experimentally ${ }^{1-5}$ and theoretically ${ }^{6-11}$ since the 1990 s with respect to the mechanism of femtosecond laser ablation. Three ablation thresholds have been identified for metals irradiated with a laser pulse of $\leq 400$ fs at a wavelength of $800 \mathrm{~nm} \cdot{ }^{12,13}$ Two of the thresholds were characterized by the electronic thermal conduction length $(\lambda \sim 80 \mathrm{~nm})$ and optical penetration length $(\delta \sim 10 \mathrm{~nm})$, respectively. ${ }^{12}$ The ablation rates at these thresholds were well expressed by the two-temperature thermal diffusion model. The third (low) ablation threshold could not be characterized by this model because the ablation rate was $\sim 0.01 \mathrm{~nm} /$ pulse (less than one atomic layer) and the threshold was strongly dependent on the laser pulse duration. ${ }^{13}$ The ablation rates were well explained by the assumption of multiphoton absorption. In order to elucidate the dynamics of the ejected particles, the velocity distribution of ions emitted from the metal by femtosecond laser ablation was measured by time-of-flight (TOF) mass spectrometry. However, the observations were limited to a laser intensity of one order of magnitude higher than the low ablation threshold since less than one ion is ejected near the threshold. Therefore, the velocity distribution could not be obtained by single-pulse laser irradiation. With regard to the laser intensity at the low ablation threshold, the absence of collisional and Coulomb effects or chemical reactions in the ablation plume is expected. Thus, the TOF velocity distribution reflects the surface dynamics of ion ejection. In the highintensity range, the emitted ions showed a double-peak structure $^{14,15}$ in the velocity distribution. The low-energy component (tens of eV) was due to thermal ions, and the high-energy component (up to several $\mathrm{keV}$ ) was produced by nonthermal processes. Such observations are quite general to metals $^{16-19}$ in this high laser intensity range. However, less attention has been paid to the ablation dynamics at the low ablation threshold. Recently, the formation of grating structures on metal surfaces has been observed. ${ }^{20}$ For fluence levels near the low ablation threshold, the grating structures had an interspace of $300 \mathrm{~nm}$, which was much shorter than the laser wavelength of $800 \mathrm{~nm}$. The interspaces of the grating structures depended on the laser fluence, and this phenomenon was well explained by the parametric decay mode ${ }^{20}$ proposed by Sakabe et al. In this model, a femtosecond laser pulse interacts with the metal and a photon in the IR region, and a plasma wave decays along the surface. The plasma wave travels slowly at less than $10^{-2}$ times the speed of light, and an ion-enriched local area appears. Before the next electron wave peak arrives, the ions experience a strong Coulomb repulsive force and can be exploded into a vacuum; in other words, a Coulomb explosion occurs. Through this process, periodic grating structures are formed. The mechanism of grating structure formation is currently under investigation. In this study, time-of-flight measurements in singlecount mode were used to investigate the dynamics of the ejected ions from the irradiated metal surface. The velocity distributions and the species of ions emitted from the metal surface were measured near the low ablation threshold. The relation between the dynamics of ion emission and grating structure formation is discussed.

\section{EXPERIMENTAL METHODS}

In the laser ablation experiments, a Gaussian-transverse mode laser beam ( $800 \mathrm{~nm}$ wavelength, $130 \mathrm{fs}$ pulse duration, and $10 \mathrm{~Hz}$ repetition rate) generated by a $T^{6}$ laser system ${ }^{21}$ was used. The laser energy was varied from 2 to $800 \mu \mathrm{J}$ by an energy attenuator. Copper of $99.9 \%$ purity was used as a target material. The size of the target was $10 \times 10 \mathrm{~mm}^{2}$, and its thickness was $1 \mathrm{~mm}$. The copper surface had a roughness of $1.5 \mathrm{~nm}$ (arithmetic mean value). Before the experiments, the target was cleaned by femtosecond laser ablation at a fluence of $0.2 \mathrm{~J} / \mathrm{cm}^{2}$. The laser beam was focused to a spot size of $50 \times 140 \mu \mathrm{m}^{2}$ on the target surface with a lens $(f$ $=30 \mathrm{~cm}$ ), at an incident angle of $70^{\circ}$ with respect to the 


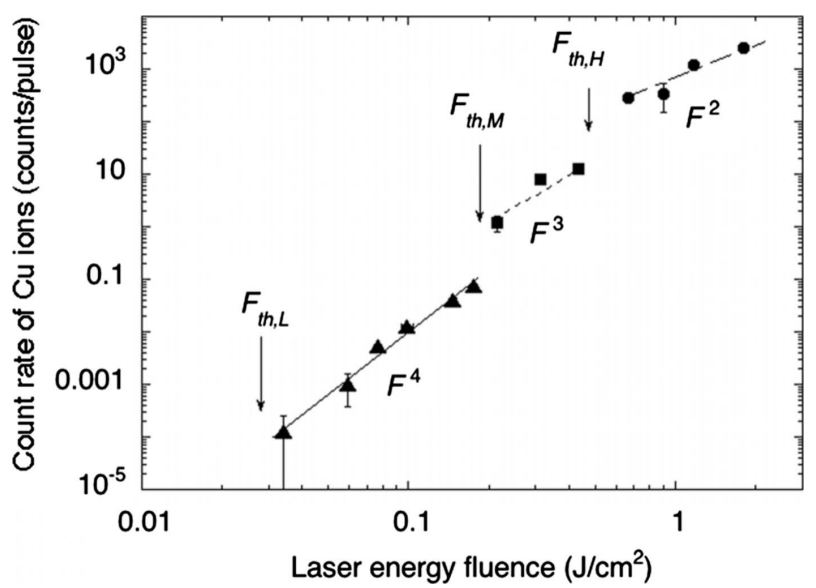

FIG. 1. Number of detected $\mathrm{Cu}$ ions as a function of laser energy fluence.

target normal. The velocity distribution of emitted ions was measured by a time-of- flight mass spectrometer with a fieldfree vacuum tube (length of $145 \mathrm{~cm}$ ) and detected by a microchannel plate. The TOF axis was normal to the copper surface. All the experiments were performed under high vacuum conditions at a pressure of $\sim 10^{-7} \mathrm{~Pa}$. The velocity distribution of ions was obtained by acquiring data for 20000 sets of single laser pulse irradiation on the $\mathrm{Cu}$ surface near the low ablation threshold. In the fluence range of $0.7-14 \mathrm{~J} / \mathrm{cm}^{2}$, the velocity distributions were obtained by averaging the signals obtained by the irradiation of three to ten laser pulses.

\section{RESULTS AND DISCUSSION}

Measurements of ion mass spectra were performed in the fluence range of $0.028-2 \mathrm{~J} / \mathrm{cm}^{2}$. Singly charged $\mathrm{Cu}$ ions were clearly detected at the fluence of $0.028 \mathrm{~J} / \mathrm{cm}^{2}$ and the number of detected ions increased with increasing laser energy fluence. Over a fluence of $1 \mathrm{~J} / \mathrm{cm}^{2}$, multiply charged ions were observed up to $Z=7$ at $2 \mathrm{~J} / \mathrm{cm}^{2}$. The distribution of charge states is likely attributable to electron-ion impact ionization. ${ }^{22}$ The count rate was defined as the number of detected $\mathrm{Cu}$ ions per laser pulse. Figure 1 shows the dependence of the count rate on the laser fluence. The experimental results for $\mathrm{Cu}$ ion emission indicate the presence of three different ablation regimes. The lowest laser fluence at which ions were emitted, referred to here as the low emission threshold $F_{t h, L}$, was $0.028 \mathrm{~J} / \mathrm{cm}^{2}$. At 0.195 and $0.470 \mathrm{~J} / \mathrm{cm}^{2}$, the ion emissions increase sharply and have a different dependence on the laser fluence. Therefore, two emission thresholds were identified at fluences of $F_{t h, M}$ $=0.195 \mathrm{~J} / \mathrm{cm}^{2}$ and $F_{t h, H}=0.470 \mathrm{~J} / \mathrm{cm}^{2}$, respectively. The dependence on the laser fluence of the number of emitted ions per laser pulse $N_{i}$ is well described as follows: $N_{i} \propto F^{4}$ for the laser fluence of $F_{t h, L}-F_{t h, M}, N_{i} \propto F^{3}$ for the laser fluence of $F_{t h, M}-F_{t h, H}$, and $N_{i} \propto F^{2}$ for the laser fluence higher than $F_{t h, H}$, where $F$ is the laser fluence. Thus, $\mathrm{Cu}$ ions produced by fluences of $F_{t h, L}-F_{t h, M}, F_{t h, M}-F_{t h, H}$, and $\geq F_{t h, H}$ are possibly the result of a nonlinear photon absorption process. The emission thresholds obtained by the present TOF

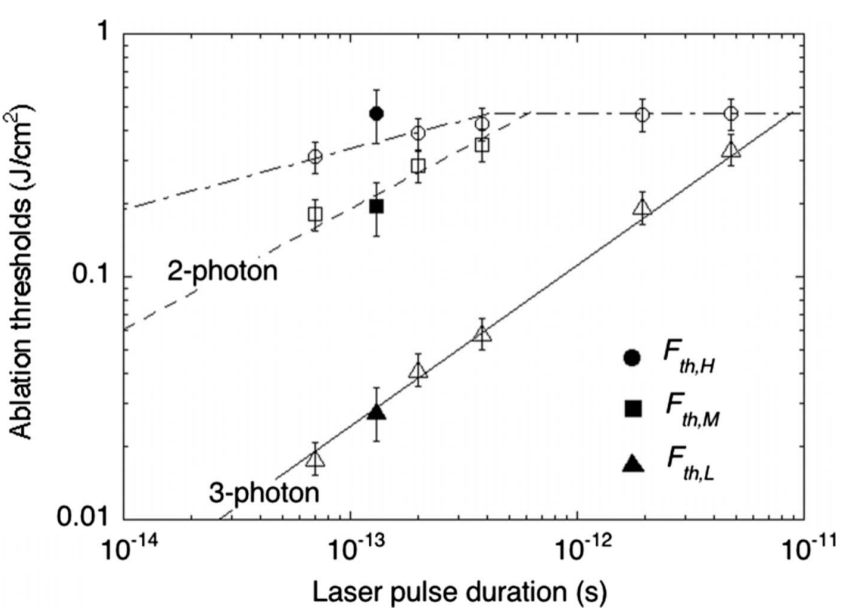

FIG. 2. Dependence of the ablation thresholds on laser pulse duration. Ion emission thresholds $(\boldsymbol{\bullet}, \mathbf{\square}$, and $\boldsymbol{\Delta})$ observed in the TOF experiment were in good agreement with ablation thresholds $(\bigcirc, \square$, and $\triangle$ ) determined by crater depth measurements reported in a previous study (Ref. 13).

experiment were of the same order of magnitude as the thresholds deduced by the crater depth measurements reported previously, ${ }^{13}$ as shown in Fig. 2. Three ablation thresholds were reported for copper, which depended on the laser pulse duration in the crater depth measurements. The thresholds are well described by ${ }^{23} F_{t h} \propto \tau_{p}^{(m-1) / m}$, where $\tau_{p}$ is the duration of the laser pulse. Within the limits of experimental error, the emission thresholds of $F_{t h, M}$ and $F_{t h, L}$ are in good agreement with the predicted thresholds under the assumption of two- and three-photon absorption processes. Therefore, $\mathrm{Cu}$ particles were ejected by $m$-photon absorption, while $\mathrm{Cu}$ ions depended on $F^{m+1}$ as shown in Fig. 1. The ionization potential of $\mathrm{Cu}$ is $6.66 \mathrm{eV},{ }^{24}$ and its work function is $4.65 \mathrm{eV}$. Therefore, at least five photons are necessary to ionize a $\mathrm{Cu}$ atom with an $800 \mathrm{~nm}$ laser $(=1.5499 \mathrm{eV})$ and four photons are necessary for multiphotoelectron ejection from a $\mathrm{Cu}$ surface. These simple considerations do not adequately explain the observed ion production with a dependence of $F^{m+1}$ over a wide fluence range. These experimental results suggest that the $\mathrm{Cu}$ ions are produced by the optical field ionization via multiphoton absorption at the sample surface. When a high-intensity laser is used to irradiate the sample surface, the bound potential of free electrons is distorted by the electric field of the laser parallel to the surface normal. This distortion of potential induces tunneling photoelectron ejection from the metal surface via multiphoton absorption. Keldysh showed the tunneling criterion for the possibility of ejecting an electron that has bounded potential, ${ }^{25}$

$$
\gamma=\frac{\nu_{L} \sqrt{2 m_{e} W}}{e E} \leq 1,
$$

where $\gamma$ is the Keldysh parameter, $\nu_{L}$ is the laser frequency, $m_{e}$ is the mass of an electron, $W$ is the work function at room temperature, $E$ is the amplitude of a laser electric field, and $e$ is the electronic charge. For an electron that absorbed $m$ photons, the work function can be reduced to $W-m h \nu_{L}$; therefore, $\gamma$ can be written as 


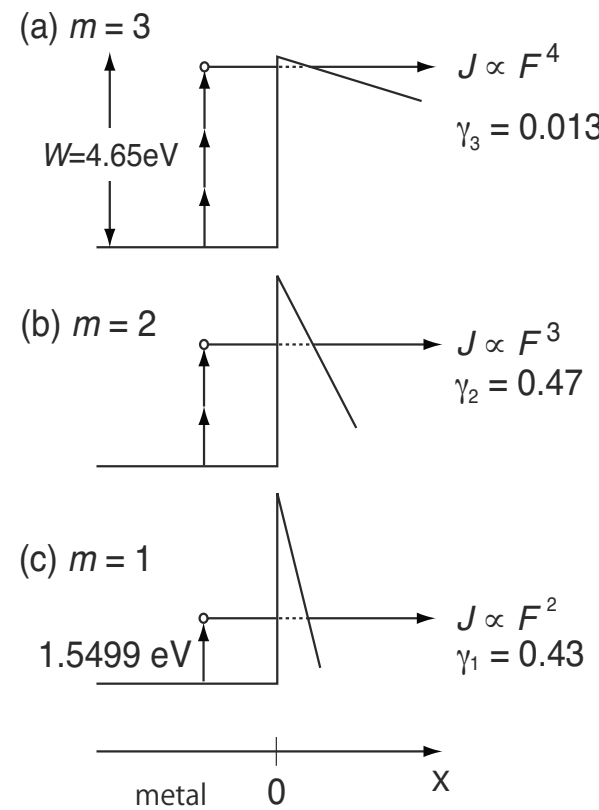

FIG. 3. Intense laser irradiation can ionize metal instantaneously by the optical field ionization via multiphoton absorption and produce metal ions. Vertical and horizontal axes show the energy level of a free electron and space $x$, respectively. $x=0$ shows the interface between the metal and vacuum. The energy level on the vacuum side is distorted by the electric field of the laser perpendicular to the surface. The distortion of the energy level (triangle shape) induces photoelectron ejection via tunneling.

$$
\gamma_{m}=\frac{\nu_{L} \sqrt{2 m_{e}\left(W-m h \nu_{L}\right)}}{e E} \leq 1,
$$

where $\nu_{L}=3.75 \times 10^{14} \mathrm{~s}^{-1}, m_{e}=9.109 \times 10^{-31} \mathrm{~kg}, W=7.449$ $\times 10^{-19} \mathrm{~J} \quad($ for $\mathrm{Cu}), \quad e=1.602 \times 10^{-19} \mathrm{C}, \quad E=1.27$ $\times 10^{9} \mathrm{~V} / \mathrm{m}$ for $F_{t h, L}=0.028 \mathrm{~J} / \mathrm{cm}^{2}, 3.36 \times 10^{9} \mathrm{~V} / \mathrm{m}$ for $F_{t h, M}=0.195 \mathrm{~J} / \mathrm{cm}^{2}$, and $5.21 \times 10^{9} \mathrm{~V} / \mathrm{m}$ for $F_{t h, H}$ $=0.470 \mathrm{~J} / \mathrm{cm}^{2}$. Under the ion emission thresholds, the tunneling criterion is satisfied as $\gamma_{3}=0.013$ for three photons, $\gamma_{2}=0.47$ for two photons, and $\gamma_{1}=0.43$ for one photon. For all $m$-photon absorptions, it is possible to eject an electron by the tunneling process shown in Fig. 3 and produce ions on the surface.

Thus, the number of ions produced is equal to the number of electrons ejected. In order to estimate the current density of ejected electrons, the Flower-Nordheim model was used. The current density of electrons from a material under an electric field applied to the surface normal can be expressed as

$$
J=\frac{A \beta^{2} E^{2}}{\phi} \exp \left(-\frac{B \phi^{2 / 3}}{\beta E}\right) \propto E^{2},
$$

where $A=1.5 \times 10^{-6}, B=6.83 \times 10^{9}, \beta$ is the field enhancement factor, $\phi$ is the bound potential, and $E$ is the applied electric field. In the present experiment, the laser was focused at an incident angle of $70^{\circ}$ relative to the $\mathrm{Cu}$ surface, and the corresponding electric field of the laser was $\sim 10^{9}-10^{10} \mathrm{~V} / \mathrm{m}$. In an electric field of this strength, the
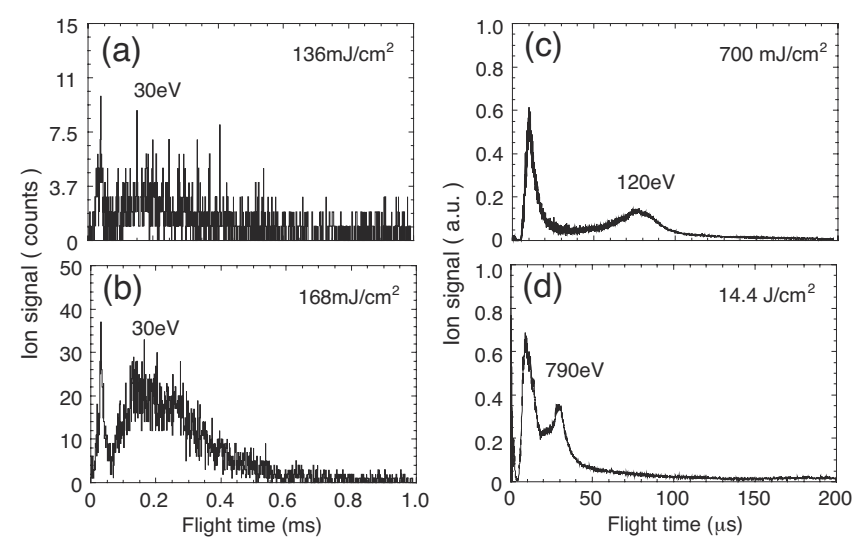

FIG. 4. Energy spectra of ions emitted from the copper sample. Most probable energies of $\mathrm{Cu}$ ions were (a) $30 \mathrm{eV}$ for $F$ $=0.136 \mathrm{~J} / \mathrm{cm}^{2}$, (b) $30 \mathrm{eV}$ for $F=0.168 \mathrm{~J} / \mathrm{cm}^{2}$, (c) $120 \mathrm{eV}$ for $F$ $=0.70 \mathrm{~J} / \mathrm{cm}^{2}$, and (d) $790 \mathrm{eV}$ for $F=14.4 \mathrm{~J} / \mathrm{cm}^{2}$.

exponential term in Eq. (3) is $\sim 1$. Therefore, the current density of ejected electrons is proportional to the laser energy fluence: $J \propto F=E^{2}$. Therefore, during tunneling photoelectron ejection from a metal surface with multiphoton absorption, ion production is expected to be dependent on $F^{m+1}$.

Figure 4 shows typical $\mathrm{Cu}$ ion TOF spectra at different laser energy fluences. No extraction field was used in these measurements. The spectra in Figs. 4(a) and 4(b) were obtained by acquiring data for 20000 sets of single pulses. The spectra in Figs. 4(c) and 4(d) were each obtained by averaging 100 laser pulses. The ions had a double-peak structure in the TOF spectra. The identity of the ionic species was confirmed by adjusting the applied voltage to an energy filter located in front of the ion detector. The fast components were hydrogen ions and the slow components were $\mathrm{Cu}$ ions. The high-energy $\mathrm{Cu}$ ions of $30 \mathrm{eV}$ were produced at $0.136 \mathrm{~J} / \mathrm{cm}^{2}$. The most probable energy of $\mathrm{Cu}$ ions linearly increased as the laser fluence increased, as shown in Fig. 5. In this paper, we classify the laser fluence into three ion emission regimes (see Fig. 1). The number of emitted ion per laser pulse $N_{i}$ was dependent on the laser fluence and in good agreement with $N_{i} \propto F^{4}$ for low fluence $(F$

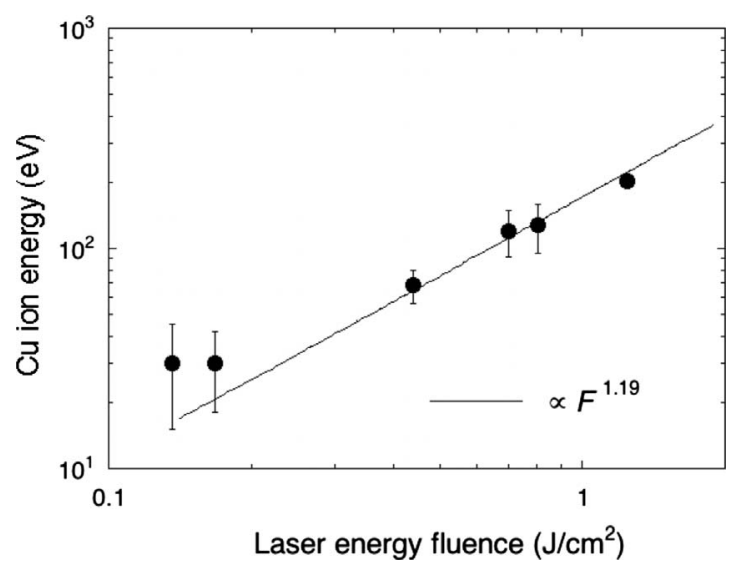

FIG. 5. Dependence of most probable energy of $\mathrm{Cu}$ ions on laser energy fluence. 
$\left.=0.028-0.195 \mathrm{~J} / \mathrm{cm}^{2}\right), \quad N_{i} \propto F^{3}$ for medium fluence $(F$ $\left.=0.195-0.47 \mathrm{~J} / \mathrm{cm}^{2}\right)$, and $N_{i} \propto F^{2}$ for high fluence $(F$ $\geq 0.47 \mathrm{~J} / \mathrm{cm}^{2}$ ). The process of ion production is well explained by multiphoton absorption and optical field ionization. The interesting $\mathrm{Cu}$ ion energy in the fluence range of $0.10-1.2 \mathrm{~J} / \mathrm{cm}^{2}$ is shown in Fig. 5. Thus, we discuss the ion energy not only at a low laser fluence but also at medium and high laser fluences.

In the fluence range of $0.10-1.2 \mathrm{~J} / \mathrm{cm}^{2}$, the energy of $\mathrm{Cu}$ ions is proportional to the laser fluence, $E \propto F^{1.19}$. This relation was analyzed within the framework of the Coulomb explosion of ions that were localized to the metal surface, and it could satisfactorily and qualitatively explain the obtained results as mentioned later. In the case of nonthermal ablation, the formation of grating structures on the $\mathrm{Cu}$ surface has been observed in this fluence range, ${ }^{20}$ and the interspaces of these grating structures are much shorter than the thermal diffusion length. Therefore, the formation of grating structures would not be observed if thermal ablation were the dominant process.

On the other hand, the dependence of $\mathrm{Cu}$ ion energy on the laser fluence was investigated separately for a laser fluence greater than $1.2 \mathrm{~J} / \mathrm{cm}^{2}$; the relation was observed experimentally to be $E \propto F^{0.5}$. This result is well explained by the emission of ions under thermal equilibrium conditions, where the most probable energy is related to the laser fluence as $E_{\text {peak }} \propto F^{0.5}$. This is in reasonable agreement with the experimental results. Thus, the ions might be produced by thermal ablation for a laser fluence greater than $1.2 \mathrm{~J} / \mathrm{cm}^{2}$. For thermal ablation at fluence greater than $1.2 \mathrm{~J} / \mathrm{cm}^{2}$, the formation of grating structures on the $\mathrm{Cu}$ surface has not been observed. $^{20}$

Therefore, the energy of the emitted ions indicates that $\mathrm{Cu}$ is ablated by a thermal process only for a laser fluence greater than $1.2 \mathrm{~J} / \mathrm{cm}^{2}$, while a nonthermal process (Coulomb explosion) is operative in the fluence range of $0.1-1.2 \mathrm{~J} / \mathrm{cm}^{2}$. In Fig. 5, the ion energy is shown, excluding ion energies for fluence greater than $1.2 \mathrm{~J} / \mathrm{cm}^{2}$, and the fluence dependence is discussed in terms of the Coulomb explosion of $\mathrm{Cu}$ ions produced by a multiphoton process and optical field ionization as shown below.

The energy of the electrons was less than $1 \mathrm{eV}$ near the low threshold fluence; such low-energy electrons were consistent with the experimental results published by other groups. ${ }^{26,27}$ No electrons corresponding to these high-energy ions were observed. Even at a laser fluence of $1 \mathrm{~J} / \mathrm{cm}^{2}$ $\left(=10^{13} \mathrm{~W} / \mathrm{cm}^{2}\right)$, the electron energy due to ponderomotive force is estimated to be only $1 \mathrm{eV}^{28}$ Therefore, the highenergy ions could not be explained by sheath acceleration of the electrons. The results of this work suggest that highenergy ions are produced by the Coulomb explosion of ions localized on the metal surface. An intense laser can ionize the metal instantaneously by the optical field ionization via multiphoton absorption and produce metal ions, which generates energetic ions by Coulomb explosion. The generation of ions from the laser-metal interaction can be identified by the energy distribution of ions emitted through the process. When the metal changes to a plasma as a result of laser irradiation, it expands adiabatically, and the emitted ions generally exhibit a Maxwellian distribution of $N_{i}$ $\sim \sqrt{E}\left(k_{B} T_{e}\right)^{(-3 / 2)} \exp \left(-E / k_{B} T_{e}\right)$, where $T_{e}$ is the electron temperature and $k_{B}$ is the Boltzmann constant. Consequently, the energy distribution has a most probable energy $E_{\text {peak }}=k_{B} T_{e}$. The electron temperature $T_{e}$ of laser-produced plasmas on the surface is proportional to the square root of the laser energy fluence: $T_{e} \propto \sqrt{F}$. Therefore, the most probable energy is related to the laser energy fluence as $E_{\text {peak }} \propto \sqrt{F}$. However, the experimental results in this fluence range did not agree well with this relation, as would be expected for the emission of ions under thermal equilibrium conditions. On the other hand, when many electrons are expelled instantaneously from an element, it becomes highly ionized. Atomic ions generated by Coulomb explosion have energy distributions of $d N / d E \sim E^{l}(l=1 / 2,0$, and $-1 / 2$ for spherical, cylindrical, and planar elements, respectively) with a finite maximum $E_{\text {max }}{ }^{29}$ Qualitatively speaking, the charge distributions due to the plasma waves that are induced by a femtosecond laser pulse are simulated by periodically arranged ion wires, and each wire exhibits a cylindrical Coulomb explosion. ${ }^{20}$ The maximum energy of $\mathrm{Cu}$ ions can be estimated as $E_{\text {max }}$ $\propto Z^{2} e^{2} n_{i} R_{0}^{2}$, where $Z$ is the ion charge state, $e$ is the electron charge, $n_{i}$ is the ion density, and $R_{0}$ is the radius of an ion cylinder. Here, $R_{0}$ is assumed to be closely related to the interspaces of the grating structures self-organized on the metal surface. ${ }^{20} R_{0}$ is determined by the interspaces of the grating structures, which depend on the laser fluence $R_{0}^{2}$ $\propto F^{0.19}$. The ion density is proportional to the density of the laser-produced plasma on the surface, as well as to the laser energy fluence $n_{i} \propto F$. Therefore, the maximum ion energy is proportional to the laser energy fluence $E_{\max } \propto F^{1.19}$. This is in reasonable agreement with the experimental results shown in Fig. 5. The experimental observations are self-consistent with the interpretation that the ions are emitted by Coulomb explosion of ions localized on the metal surface by an intense femtosecond laser pulse.

\section{CONCLUSION}

In conclusion, femtosecond laser ablation of $\mathrm{Cu}$ by shortpulse laser irradiation ( $800 \mathrm{~nm}$ and $130 \mathrm{fs}$ ) was studied in the laser energy fluence range of $0.028-14.4 \mathrm{~J} / \mathrm{cm}^{2}$. In order to elucidate the dynamics of the ejected particles, the energy distribution of ions emitted from the metal with femtosecond laser ablation was measured by time-of-flight mass spectrometry. Three thresholds for ion emission were identified. The number of emitted ions per laser pulse $N_{i}$ was dependent on the laser fluence and was in good agreement with $N_{i}$ $\propto F^{4}$ for the laser fluence of $F_{t h, L}-F_{t h, M}, N_{i} \propto F^{3}$ for the laser fluence of $F_{t h, M}-F_{t h, H}$, and $N_{i} \propto F^{2}$ for $\geq F_{t h, H}$. The process of ion production is well explained by multiphoton absorption and optical field ionization. High-energy $\mathrm{Cu}$ ions of 30 $\mathrm{eV}$ were produced at a low laser fluence of $0.136 \mathrm{~J} / \mathrm{cm}^{2}$. The most probable energy of $\mathrm{Cu}$ ions increased as the laser energy fluence increased. The experimental results were analyzed within the framework of the Coulomb explosion of ions that were localized to the metal surface, which could satisfactorily and qualitatively explain the obtained results. 


\section{ACKNOWLEDGMENTS}

This study was financially supported by a Grant-in-Aid for Scientific Research (A) (Grant No. 18206006) from the Ministry of Education, Culture, Sports, Science and Technology (MEXT), Japan, the Research Foundation of the Murata
Science Foundation, and the Amada Foundation for Metal Work Technology and partially supported by a Grant-in-Aid for the Global COE Program "The Next Generation of Physics, Spun from Universality and Emergence" from MEXT, Japan.
${ }^{1}$ J. G. Fujimoto, J. M. Liu, E. P. Ippen, and N. Bloembergen, Phys. Rev. Lett. 53, 1837 (1984).

${ }^{2}$ A. M. Komashko, M. D. Feit, A. M. Rubenchik, M. D. Perry, and P. S. Banks, Appl. Phys. A: Mater. Sci. Process. 69, S95 (1999).

${ }^{3}$ S. Amoruso, X. Wang, C. Altucci, C. deLisio, M. Armenante, R. Bruzzese, and R. Velotta, Appl. Phys. Lett. 77, 3728 (2000).

${ }^{4}$ B. Rethfeld, K. Sokolowski-Tinten, D. von der Linde, and S. I. Anisimov, Phys. Rev. B 65, 092103 (2002).

${ }^{5}$ V. Margetic, T. Ban, F. Leis, K. Niemax, and R. Hergenroder, Spectrochim. Acta, Part B 58, 415 (2003).

${ }^{6}$ E. G. Gamaly, N. R. Madsen, M. Duering, A. V. Rode, V. Z. Kolev, and B. Luther-Davies, Phys. Rev. B 71, 174405 (2005).

${ }^{7}$ M. E. Povarnitsyn, T. E. Itina, M. Sentis, K. V. Khishchenko, and P. R. Levashov, Phys. Rev. B 75, 235414 (2007).

${ }^{8}$ T. E. Itina, Chem. Phys. Lett. 452, 129 (2008).

${ }^{9}$ Y. Hirayama, P. A. Atanasov, M. Obara, N. N. Nedialkov, and S. E. Imamova, Jpn. J. Appl. Phys., Part 1 45, 792 (2006).

${ }^{10}$ A. K. Upadhyay, N. A. Inogamov, B. Rethfeld, and H. M. Urbassek, Phys. Rev. B 78, 045437 (2008).

${ }^{11}$ D. S. Ivanov and B. Rethfeld, Appl. Surf. Sci. 255, 9724 (2009).

${ }^{12}$ S. Nolte, C. Momma, H. Jacobs, A. Tunnermann, B. N. Chichkov, B. Wellegehausen, and H. Welling, J. Opt. Soc. Am. B 14, 2716 (1997)

${ }^{13}$ M. Hashida, A. F. Semerok, O. Gobert, G. Petite, Y. Izawa, and J. F. Wagner, Appl. Surf. Sci. 197-198, 862 (2002).

${ }^{14}$ S. Amoruso, X. Wang, C. Altucci, C. de Lisio, M. Armenante, R. Bruzzese, N. Spinelli, and R. Velotta, Appl. Surf. Sci. 186, 358 (2002).
${ }^{15}$ Y. Okano, H. Kishimura, A. Yazaki, F. Saito, Y. Hironaka, K. G. Nakamura, K. Kondo, and Y. Oguri, J. Surf. Sci. Soc. Jpn. 22, 438 (2001).

${ }^{16}$ M. Ye and C. P. Grigoropoulos, J. Appl. Phys. 89, 5183 (2001).

${ }^{17}$ S. Bashir, M. S. Rafique, and W. Husinsky, Appl. Surf. Sci. 255, 8372 (2009).

${ }^{18}$ H. Dachraoui, W. Husinsky, and G. Betz, Appl. Phys. A: Mater. Sci. Process. 83, 333 (2006).

${ }^{19}$ H. Dachraoui and W. Husinsky, Appl. Phys. Lett. 89, 104102 (2006).

${ }^{20}$ S. Sakabe, M. Hashida, S. Tokita, S. Namba, and K. Okamuro, Phys. Rev. B 79, 033409 (2009).

${ }^{21}$ S. Tokita, M. Hashida, S. Masuno, S. Namba, and S. Sakabe, Opt. Express 16, 14875 (2008).

${ }^{22}$ M. Gryziński, Phys. Rev. 138, A336 (1965).

${ }^{23}$ S. Preuss, M. Spath, Y. Zhang, and M. Stuke, Appl. Phys. Lett. 62, 3049 (1993).

${ }^{24}$ T. A. Carlson, C. W. Nestor, Jr., N. Wasserman, and J. D. McDowell, At. Data 2, 63 (1970).

${ }^{25}$ L. V. Keldysh, Sov. Phys. JETP 20, 1307 (1965).

${ }^{26}$ M. Aeschlimann, C. Schmuttenmaer, H. Elsayed-Ali, R. Miller, J. Cao, Y. Gao, and D. Mantell, J. Chem. Phys. 102, 8606 (1995).

${ }^{27}$ D. Riffe, X. Wang, M. Downer, D. Fisher, T. Tajima, and J. Erskine, J. Opt. Soc. Am. B 10, 1424 (1993).

${ }^{28}$ M. Hirokane, S. Shimizu, M. Hashida, S. Okada, S. Okihara, F. Sato, T. Iida, and S. Sakabe, Phys. Rev. A 69, 063201 (2004).

${ }^{29}$ S. Sakabe, K. Shirai, M. Hashida, S. Shimizu, and S. Masuno, Phys. Rev. A 74, 043205 (2006). 